\title{
I 024 A novel CMR-based myocardial salvage index predicts left ventricular remodeling after acute myocardial infarction: insights from T2-weighted and late gadolinium enhancement imaging Hassan Abdel-Aty*, Anja Zagrosek, Ralf Wassmuth, Steffen Bohl, Philipp Boyé, Andre Rudolph, Daniel Messroghli, Wolfgang Utz, Rainer Dietz and Jeanette Schulz-Menger
}

Address: Franz-Volhard-Klinik, Charité Universitätsmedizin Berlin, Helios-Klinikum Berlin, Berlin, Germany

* Corresponding author

from I th Annual SCMR Scientific Sessions

Los Angeles, CA, USA. I-3 February 2008

Published: 22 October 2008

Journal of Cardiovascular Magnetic Resonance 2008, I0(SuppI I):AI49 doi:I0.I I86/I532-429X-I0-SI-AI49

This abstract is available from: http://jcmr-online.com/content/I0/SI/AI49

(C) 2008 Abdel-Aty et al; licensee BioMed Central Ltd.

\section{Background}

Late gadolinium enhancement (LGE) accurately identifies irreversible injury after acute myocardial infarction (AMI). T2-weighted imaging has the ability to quantify the area at risk in the same setting. We investigated the value of a novel myocardial salvage index based on the difference between the extents of T2-weighted abnormality and LGE to predict long-term left ventricular remodeling after AMI.

\section{Methods}

We enrolled 41 patients ( 31 males, $56 \pm 11$ y) $3 \pm 2$ days after successful infarct reperfusion by PCI. Thirty-five patients returned for follow up $15 \pm 13$ months (median 12 months) after the acute event. The CMR protocol consisted of cine steady state free precession, triple inversion recovery $\mathrm{T} 2$-weighted and late gadolinium enhancement imaging (10 minutes after the iv injection of gadoliniumDTPA). Left ventricular volumes and function were quantified independent from the tissue characterization analysis and by different observers. In the acute phase, T2weighted and LGE images were analyzed by special software (CMR42R, CIRCLE, Calgary) to quantify volumes of abnormal signal, which were expressed as a percent of the total LV volume. The difference between T2 and LGE extents (T2-LGE) was then calculated as a measure of myocardial salvage. The primary end-points were the change in end-diastolic volume (delta-EDV) and endsystolic volume (delta-ESV) over time.

\section{Results}

T2-weighted abnormalities were detected only during the acute phase. T2-LGE but not LGE alone ( $\mathrm{p}=0.1)$ correlated with the time to reperfusion $(\mathrm{r}=-0.39 ; \mathrm{p}=0.014)$. Both T2-LGE $(\mathrm{r}=-0.52 ; \mathrm{p}=0.002)$ and LGE alone $(\mathrm{r}=$ $0.41 ; \mathrm{p}=0.018)$ correlated with delta EDV. T2-LGE correlated with delta-ESV $(r=-0.39, \mathrm{p}=0.026)$ but LGE alone $\operatorname{did} \operatorname{not}(\mathrm{p}=0.43)$.

The relation between T2-LGE, delta EDV and delta-ESV remained significant after correcting for the inter-study duration, time to reperfusion, ejection fraction and LGE extent.

\section{Conclusion}

We propose a myocardial salvage index based on the difference between T2-weighted abnormality and late gadolinium enhancement. This novel CMR-based parameter may have the potential to serve as a surrogate end-point in studies assessing the efficacy of infarct reperfusion strategies. 